\title{
Du pessimisme à l'optimisme \\ Les métamorphoses de la pensée raciale au Brésil et la réception des théories de Gilberto Freyre (1890-1930)
}

Les théories raciales constituent un aspect mal étudié des »transferts culturels«, un champ de recherche pourtant fort à la mode. Dans cette perspective, le Brésil doit occuper une place centrale dans la mesure où la »question raciale«, même si ce n'est jamais exprimé aussi crûment, est conçue comme le problème national par excellence entre le milieu du XIX ${ }^{\mathrm{e}}$ siècle et les années 1930. La présence de Noirs au Brésil découle directement de la traite négrière: ce pays a reçu, entre 1500 et 1850 (interdiction effective du trafic), quatre millions d'Africains, soit un tiers du total d'esclaves destinés aux Amériques ${ }^{1}$.

Les descriptions rapportées du Brésil par les voyageurs ont également fourni bien des matériaux aux théoriciens des races, quand ces derniers n'y ont pas eux-mêmes séjourné. Arthur de Gobineau, diplomate français à Rio pendant un an et demi en 18691870, voit ainsi la confirmation de »'inégalité des races humaines«, postulat de son ouvrage publié en 1853; Louis Agassiz (1807-1873), naturaliste suisse installé aux États-Unis, adversaire de Darwin et défenseur du polygénisme, constate au Brésil la supériorité de la race blanche entre avril 1865 et juillet $1866 . \mathrm{Du} \mathrm{XVI}^{\mathrm{e}}$ au XXI ${ }^{\mathrm{e}}$ siècle, de Montaigne à l'apologie postmoderne du métissage, le Brésil fait figure de laboratoire de l'humanité, de modèle d'observation ou d'imitation, de lieu de fixation de bien des fantasmes².

$\mathrm{Au}$ tournant des $\mathrm{XIX}^{\mathrm{e}}$ et $\mathrm{XX}^{\mathrm{e}}$ siècles, les Brésiliens importent et adaptent la pensée raciale venue d'Europe, principalement de France. Les difficultés du Brésil à devenir une puissance sont attribuées à son peuplement. Au début des années 1930, le Brésil devient à son tour producteur d'une pensée raciale promise à un bel avenir international, celle de Gilberto Freyre (1901-1987), l'un des intellectuels les plus influents du $\mathrm{XX}^{\mathrm{e}}$ siècle sur le plan mondial.

1 À noter que 1,7 million d'esclaves entrent au Brésil dans la première moitié du XIX ${ }^{\mathrm{e}}$ siècle. Luiz Felipe de AlENCASTRO, O trato dos viventes. Formação do Brasil no Atlântico Sul, São Paulo 2000, p. 69.

2 Cf. l'ultime phrase de l'»Histoire du Brésil« de Bartolomé Bennassar et Richard Marin: »en terme de vitalité, de génie d'un peuple ou de melting pot des couleurs, on aimerait que le monde de demain ressemblât au Brésil«, dans: Bartolomé BENNASSAR, Richard MARIN, Histoire du Brésil 1500-2000, Paris 2000, p. 526. 


\section{LE PAYS QUI VOULAIT ÊTRE BLANC}

En 1889, la république est proclamée au Brésil et, avec elle, le principe d'égalité. L'esclavage, aboli l'année précédente par la monarchie, est rituellement condamné en paroles, mais rien n'est fait pour que les anciens esclaves et leurs descendants accèdent à la plénitude de leur citoyenneté nouvelle. Aucune mesure juridique n'établit formellement une colour bar entre les citoyens, mais aucune disposition ne vient émanciper socialement les ex-captifs qui constituent la part la plus misérable de la population. Le suffrage universel a été institué par la république, mais les analphabètes (qui ne sont pas tous noirs ou métis) - presque $90 \%$ de la population - en sont exclus.

D'après le recensement de 1890 , les Blancs représentent $44 \%$ d'une population évaluée à quatorze millions d'individus, les Noirs, $14,6 \%$ et les métis, $41,4 \% \%^{3}$. D'après une idée bien ancrée dans les esprits depuis la période de l'Indépendance (1822), le pays ignorerait le préjugé contre la couleur de la peau. La carrière de quelques métis au sein de l'administration impériale ou dans les arts et lettres est régulièrement citée pour étayer cette affirmation, relayée par les observations des étrangers.

À partir des années 1880 , le tableau a singulièrement évolué. Les classes dirigeantes brésiliennes ont une vision de leur société qui s'apparente à un déni: le Brésil est un pays blanc, engagé sur la voie de la civilisation. Une vulgate raciste, nourrie aux écrits de Georges Vacher de Lapouge ou de Gustave Le Bon, puis de Herbert Spencer, achève de convaincre que les Noirs et les Indiens appartiennent à des »races« inférieures ou attardées, incompatibles avec la civilisation à laquelle aspirent les élites dirigeantes. Il est difficile, voire impossible, de les admettre comme une partie intégrante de la nation et surtout, de laisser l'image d'un Brésil non-blanc s'imposer à l'extérieur. En 1911, le directeur du Musée national, João Batista Lacerda, reçoit de vives critiques pour avoir présenté à Londres, au congrès universel des races, un mémoire où il laissait entendre que les Blancs n'étaient pas majoritaires au Brésil4. Quant au président Epitácio Pessoa (1919-1922), il interdit aux joueurs de couleur de jouer dans l'équipe nationale de footballs.

Si les indigènes suscitent depuis longtemps de l'intérêt intellectuel, celui-ci est tardif concernant les descendants des Africains, négligés par les rares institutions scientifiques du Brésil jusqu'aux années 1890. Celles-ci étaient au nombre de cinq, le Musée national (fondé en 1808), les facultés de médecine de Salvador de Bahia et de Rio de Janeiro, créées la même année, et les musées Paraense (Goeldi), à Belém du Pará en Amazonie (1866), et Paulista (1893), auxquels il faut ajouter l'Institut historique et géographique brésilien (1838) et les sociétés savantes qui se développent sur le modèle de celui-ci dans les différents États de la fédération sous la république. Aucune de ces institutions n'avait jugé utile de collectionner les objets produits par les AfroBrésiliens ni de mesurer les crânes de ces derniers ou d'examiner leur langage. Les

3 Lília Moritz Schwarcz, O espetáculo das raças. Cientistas, instituições e questão racial no Brasil, 1870-1930, São Paulo 1993, p. 251.

4 João Batista LACERDA, O congresso universal das Raças, s.l. 1912.

5 Nosso Século 1910/1930. Anos de crise e criação I, São Paulo 1980, p. 7. 
premiers travaux les concernant sont l'œuvre d'un médecin bahianais, Raimundo Nina Rodrigues (1862-1906) à la fin du sièclé6.

Le morcellement à l'infini de l'espèce humaine en »races« et la stricte hiérarchisation de celles-ci n'en finissent pas de poser des problèmes insurmontables aux classes dirigeantes brésiliennes. Les doctrines qui placent l'aryen à la tête des »races« assombrissent l'avenir des contrées dominées par les »races latines«, comme le Brésil. Dans ce très vaste pays, les théories raciales accentuent les différences entre les grandes régions et participent à la justification du leadership des États méridionaux sur ceux du Nord, peuplés majoritairement de non-Blancs. En 1887, un républicain paulista, Alberto Sales, publie un opuscule, très imprégné de spencérisme, en faveur de la séparation de sa province de l'empire du Brésil. L'un des arguments qui légitime l'émancipation de São Paulo tient à son peuplement. D'après Sales, la »race blanche« qui s'est installée dans la région au $\mathrm{XVI}^{\mathrm{e}}$ siècle était d'une souche beaucoup plus noble et plus pure que celle qui a conquis le Nord. De plus, à São Paulo et au contraire de ce qui advint dans le Nord, les colons blancs »composés d'éléments purs ne se mélangeaient pas avec les indigènes « ${ }^{7}$. En conclusion, Alberto Sales explique les raisons du dynamisme pauliste:

on peut dire que le Brésil se divise ethnologiquement en trois grandes régions: le Nord, le Centre, le Sud. Dans la première, par croisement, c'est le sang indigène qui a prédominé; dans la deuxième, le sang africain; dans la troisième, le sang blanc. Ou, selon un écrivain brésilien contemporain: >sur toute la superficie du Brésil, le type blanc l'a emporté. Mais si nous distinguons la part de chaque race dans ces croisements, il est possible d'obtenir la division suivante: les populations du nord du Brésil jusqu'au Pernambouc possèdent une marque fortement indigène; de Bahia jusqu'aux provinces de Rio de Janeiro et du Minas Gerais, le sang africain s'est insinué sur une large échelle; de là vers le Sud, les populations apparaissent moins mélangées<. Voilà pourquoi São Paulo est devenu aujourd'hui le centre d'un remarquable développement moral et intellectuel 8 .

Plus encore que la question des »races«, c'est celle de leur métissage qui occupe toutes les attentions et plonge les élites brésiliennes dans la plus angoissante perplexité.

\section{LE MÉTISSAGE, MALÉDICTION OU MAL TRANSITOIRE?}

Entre les Noirs et les Blancs s'est, de toute évidence, immiscé depuis longtemps une catégorie intermédiaire, celle des métis (mestiço) et plus particulièrement celle des mulatos (mulâtres). L'étymologie de »mulâtre«, forgé à partir de »mulet«, rend assez bien compte du problème et de son actualisation par les théories raciales à un moment où les statistiques montrent que les mulatos sont au moins aussi nombreux que les

6 Thomas E. Skidmore, Black into White. Race and Nationality in Brazilian Thought, Durham, Londres 1993, p. 57.

7 Alberto SAles, A Pátria paulista, Brasília 1983 ( $1^{\text {re }}$ édition 1887), p. 103. Alberto Sales est le frère de Manuel Campos Sales, président de la République entre 1898 et 1902.

8 Ibid., p. 104. 
Blancs dans la population brésilienne. Le mulâtre, produit du croisement avec une »race inférieure «, ne peut être qu'un type dégénéré.

En 1894, Raimundo Nina Rodrigues, médecin de la faculté de Bahia, classe et hiérarchise les métis en trois catégories qui vont du »supérieur« au dégénéré. En vertu de ce classement, il propose que les Noirs échappent à toute responsabilité pénale, comme des mineurs, et que les métis aient une responsabilité limitée devant la loi en fonction de leurs capacités. La meilleure solution pour l'avenir de la »race« reste, en tout cas, d'éviter le métissage9.

Le directeur du Musée national - la principale institution scientifique du pays -, João Batista Lacerda, a des perspectives plus optimistes sur la question. Il a présenté à Londres, au congrès universel des races de 1911, un mémoire intitulé "Les métis au Brésil«, qui postule, en définitive, le blanchiment à terme de la population brésilienne. Selon les conjectures de Lacerda, en 2012, les Noirs auront totalement disparu du Brésil et les Blancs formeront alors $80 \%$ de la population. Le cinquième restant sera le fait des indigènes (estimés à $17 \%$ en 2012 ) et de $3 \%$ résiduels de »métis négroïdes « 10 . La disparition à brève échéance des Noirs du Brésil est une idée très largement partagée entre les années 1890 et 1910 . On en veut pour preuve le solde naturel très négatif qu'avancent les statisticiens. Pour Lacerda (et d'autres auteurs), le Brésil règle en douceur un problème qui menace en revanche la stabilité des États-Unis. Aux ÉtatsUnis, indique Lacerda, les Noirs sont organisés. Ils possèdent leurs institutions et forment en quelque sorte un État dans l'État. Rien de tel au Brésil où:

sans aucun lien les unissant, sans aucune espèce d'initiative, perdus sur des chemins impraticables comme les animaux égarés d'un troupeau, les Noirs n'ont pas pu trouver jusqu'à aujourd'hui une orientation pour se diriger vers une quelconque organisation sociale. L'abandon, l'isolement, l'inaction, l'incurie auxquels ils se sont livrés depuis l'abolition de l'esclavage, ont augmenté chaque fois plus leur décadence et concourent à leur extinction. Au Brésil, le problème de la race noire se résout sans difficulté, alors qu'aux États-Unis se profile encore aujourd'hui pour les hommes d'État de ce pays un problème insoluble, cerné de difficultés et de dangers ${ }^{11}$.

Les mulatos aussi sont voués à l'extinction progressive. En trois générations, d'après Lacerda, les caractères négroïdes sont pratiquement effacés. Le blanchiment, grâce aussi à l'immigration européenne massive - entre 1870 et 1930 , deux millions de migrants s'installent au Brésil -, est l'espoir auquel s'accroche la majeure partie des classes dirigeantes.

\section{LE MÉTISSAGE ET LA CIVILISATION BRÉSILIENNE}

Quelques individus isolés s'écartent cependant de la doxa du blanchiment dans les années 1910. Manoel Bonfim (1868-1932), un médecin originaire du nord du Brésil,

9 SKIDMORE, Black into White (voir n. 6), p. 60.

10 LACERDA, O congresso universal das Raças (voir n. 4), p. 97.

11 Ibid. 
rompt même avec les explications raciales et se penche sur les causes historiques de ce qu'il appelle les »maux de l'Amérique latine «12. Pour Bonfim, c'est le pillage colonial, le »parasitisme des métropoles«, et non la prétendue infériorité des populations locales, qui a entravé pour longtemps l'essor de la région. Un homme politique de l'État de Rio, Alberto Torres, est l'une des rares voix à remettre en cause le bien-fondé de la politique migratoire, de l'immigrant perçu comme un facteur de civilisation et à inviter les pouvoirs publics à soutenir les »travailleurs nationaux«, c'est-à-dire les ouvriers agricoles noirs ou métis pour la plupart13. Bonfim, comme Torres, connaîtra le succès dans les années 1930. Car, même si une minorité d'auteurs se convainc - parfois à l'aide de l'œuvre de Franz Boas - que les conditions sociales pèsent plus que le biologique, Francisco Oliveira Viana (1883-1951), intellectuel renommé et ferme propagateur de théories racistes, se félicite encore du blanchiment de la population dans son commentaire du Recensement général de la population de $1920^{14}$.

Le changement de paradigme dominant a lieu à partir de 1933 avec la publication de »Casa Grande \& Senzala« (»Maîtres et esclaves. La formation de la société brésilienne«) de Gilberto Freyre. Lui, qui s'est toujours présenté comme sociologue, est originaire du Pernambouc, de ce Nord-Est dont certains intellectuels interprètent le déclin comme le résultat de son peuplement bigarré. En tant qu'auteur, Freyre a été une figure de proue du mouvement culturel régionaliste qui a cherché à s'affirmer dans les années 1920 par rapport au Sud. Freyre a une autre caractéristique qui le distingue de beaucoup de ses collègues écrivains et intellectuels: il a fait ses études supérieures aux États-Unis, au Texas d'abord, puis à Columbia, où il a soutenu une thèse sur la société brésilienne au XIX ${ }^{\mathrm{e}}$ siècle. Lui-même a souligné l'importance qu'avaient eue sur son travail ses contacts avec Franz Boas, même si la nature de cette influence fait aujourd'hui l'objet de discussions savantes ${ }^{15}$. Après la révolution de 1930, Freyre s'exile quelques temps, notamment à Stanford, où il rédige son maître ouvrage: „Casa Grande \& Senzala«.

Ce livre est devenu le classique des classiques de la littérature nationale, le livre que tout honnête Brésilien se doit d'avoir lu. C'est aussi le premier titre d'une œuvre pléthorique dont la publication s'étale sur une cinquantaine d'années. „Casa Grande \& Senzala« est la réponse de Freyre aux interrogations de sa génération, mais aussi des générations précédentes, sur la lancinante question du métissage. Dans la préface à la première édition du livre, Freyre raconte:

Je vis une fois, après trois longues années d'absence du Brésil, une bande de marins mulâtres et métis, descendant les rues pleines de neige de Brooklyn. Ils me donnèrent l'impression de caricatures. Il me vint à l'esprit une phrase d'un voyageur américain, que je venais de lire sur le Brésil:

12 Manuel BonfiM, A América latina. Males de origem, Rio de Janeiro 1905.

13 Alberto TORRES, O Problema Nacional Brasileiro: introdução a um programa de organização nacional, São Paulo 1982 ( $1^{\text {re }}$ édition 1914).

14 Francisco José Oliveira VIANA, »Evolução da raça«. Recenseamento do Brazil, Rio de Janeiro 1922, p. 313-344.

15 Maria Lúcia Pallares-Burke, Gilberto Freyre, um Vitoriano nos Trópicos, São Paulo 2005. 
l'aspect effrayant de dégénérés de presque tous ses habitants. Voilà donc le résultat de la miscigénation 16 !

D'après Freyre, ce fut Boas qui l'aida à opérer sa révolution copernicienne:

J'appris à considérer comme fondamentale la distinction entre race et civilisation, à séparer les effets des relations purement génétiques des influences sociales, de l'héritage culturel et du milieu. Tout mon livre repose sur cette distinction. Comme sur la distinction entre l'hérédité raciale et l'hérédité familiale17.

Freyre n'annule en rien le facteur racial, sous-jacent dans son œuvre, mais son intérêt principal porte sur les conditions sociales et les aspects culturels. Il manifeste surtout une curiosité inédite pour la population noire du Brésil et l'histoire de ses pratiques, alors que seuls les Indiens avaient monopolisé jusqu'alors l'attention des érudits. Enfin, Gilberto Freyre réhabilite le colonisateur décrié, le Portugais, auquel on attribuait volontiers les maux historiques du Brésil. Pour le sociologue pernamboucain, les Portugais, mieux prédisposés à la colonisation que les autres Européens, ont été les seuls à faire surgir une civilisation originale sous les tropiques. Le métissage et l'appropriation par les Portugais d'usages culturels des populations natives ou africaines expriment ce génie lusitain de l'adaptation dont la civilisation patriarcale des plantations du Nordeste brésilien est la quintessence. Freyre écrit des phrases qui font un effet révolutionnaire dans le Brésil de 1933:

Les esclaves venus des aires culturelles africaines les plus avancées furent un élément actif, créateur, et on pourrait dire noble de la colonisation du Brésil, abaissés seulement par leur condition d'esclaves. Loin de n'avoir été que des animaux de traction et des ouvriers agricoles, ils exercèrent une fonction civilisatrice ${ }^{18}$.

La place de la sexualité dans »Casa Grande \& Senzala« - le thème est au cœur de deux des cinq chapitres du livre - a aussi suscité l'émoi et classé le distingué Gilberto Freyre dans la catégorie des pornographes.

Les thématiques de »Casa Grande \& Senzala« sont cependant filées par toutes sortes de polygraphes au service de la dictature de l'Estado Novo, instaurée en 1937. Très officiellement, le Brésil devient alors un »pays métis«, une synthèse unique au monde, un parangon de tolérance raciale. Le métissage est désormais conciliable avec un avenir radieux. Après 1945, l'Unesco érige Gilberto Freyre en maître à penser de la lutte contre le racisme, mais, fidèle au noyau dur de sa pensée, ce dernier s'en va exalter la supériorité portugaise en matière de colonisation, alors que soufflent les vents de la décolonisation. La dictature portugaise dirigée par António de Oliveira Salazar se convertit aux vertus du métissage et organise pour Gilberto Freyre une grande tournée

16 Gilberto FreYre, Maîtres et esclaves. La formation de la société brésilienne, Paris 1974, p. 435. La première édition française de »Casa Grande \& Senzala« date de 1952 et est précédée d'une préface de Lucien Febvre.

17 Ibid., p. 435.

18 Ibid., p. 289. 
à travers l'empire colonial en 1951. À cette occasion, lors d'une conférence prononcée à Goa, aux portes de l'Inde de Nehru, Freyre lance le concept de »lusotropicalisme«, dont le Portugal de Salazar va faire son miel pour justifier l'inaliénabilité de son domaine colonial. L'idéologie lusotropicale n'a pas été balayée par la révolution des Eillets et les indépendances de l'Afrique portugaise. Elle a débordé de la propagande salazariste pour toucher un vaste public, convaincu du génie assimilateur du Portugal et empreint de bonne conscience paternaliste ${ }^{19}$.

Ainsi, Gilberto Freyre a fortement contribué à la formulation de deux idéologies nationales, élaborées à des périodes différentes au Brésil, puis au Portugal. Ce sont les mêmes idées qui ont présidé à la création en 1996 de la Communauté des pays de langue portugaise. Si Gilberto Freyre, très critiqué au Brésil dans les années 1970, est actuellement une icône à la fois intellectuelle (pour toute une école historique, notamment) et nationale, le modèle freyrien est toutefois vivement contesté. Il devrait l'être d'abord en tant que pensée raciale, puisque le métissage s'inscrit délibérément dans une telle logique. Il est dénoncé surtout comme un tour de passe-passe qui a longtemps servi à escamoter la réalité de la discrimination raciale au Brésil. La remise en cause des théories freyriennes passe notamment par l'émergence de nouvelles théories raciales qui dénoncent le métissage comme une forme de négation de la présence et de la force des Noirs au Brésil. Le métissage fait de nouveau problème. Avec cette démarche, en effet, le mot »métis« retrouve en somme le sens usuel qui était le sien jusqu'au début du $\mathrm{XX}^{\mathrm{e}}$ siècle: »métis« renvoyait plus à la couleur foncée de la peau qu'à l'idée de mélange. Ainsi, après avoir été menacés de disparition par blanchiment, les métis brésiliens courent désormais le risque inverse.

19 Voir Yves LÉONARD, Immuable et changeant, le lusotropicalisme au Portugal, dans: Arquivos do Centro Cultural Calouste Gulbenkian XLII (2001), p. 107-117. 